\title{
Microgram per Milliliter per Meter Squared
}

National Cancer Institute

\section{Source}

National Cancer Institute. Microgram per Milliliter per Meter Squared. NCI Thesaurus.

Code C119511.

A unit of concentration equal to micrograms per milliliter, divided by meters squared. 